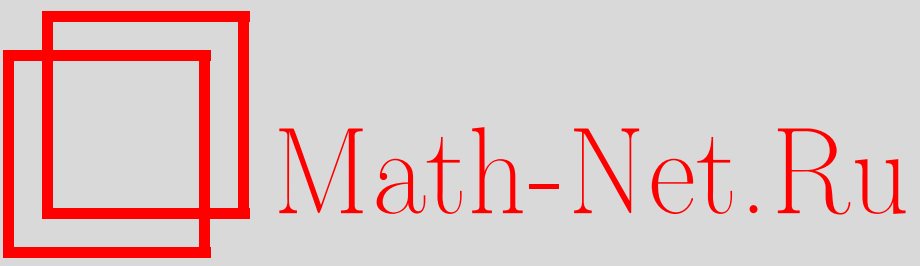

Дж. Харнад, А. Ю. Орлов, Скалярные произведения симметрических функций и матричные интегралы, ТМФ, 2003, том 137, номер 3, 375-392

DOI: https://doi.org/10.4213/tmf279

Использование Общероссийского математического портала Math-Net.Ru подразумевает, что вы прочитали и согласны с пользовательским соглашением

http://www.mathnet.ru/rus/agreement

Параметры загрузки:

IP: 3.85 .7 .115

26 апреля 2023 г., 15:14:55 
ТЕОРЕТИЧЕСКАЯ

И МАТЕМАТИЧЕСКАЯ

ФИЗИКА

Том 137, № 3

декабрь, 2003

(C) 2003 г. Дж. Харнад А. Ю. Орлов ${ }^{\dagger}$

\title{
СКАЛЯРНЫЕ ПРОИЗВЕДЕНИЯ СИММЕТРИЧЕСКИХ ФУНКЦИЙ И МАТРИЧНЫЕ ИНТЕГРАЛЫ
}

\begin{abstract}
Представлены соотношения между билинейными операторами типа Хироты, скалярными произведениями на пространствах симметрических функций и интегралами, задающими статистические суммы матричных моделей. Используя представление фермионного пространства Фока, удается доказать разлагаемость ассоциированного класса тау-функций $\tau_{r, n}$ иерархии Кадомцева-Петвиашвили и двумеризованной цепочки Тоды в ряд по функциям Шура, обобщающий разложение в гипергеометрический ряд и связанный с формулами скалярного произведения. Показано, как специально выбранные тау-функции из такого класса отождествляются как формальные ряды со статистическими суммами. Выведено в замкнутой форме разложение $\ln \tau_{r, n}$ в терминах функций Шура.
\end{abstract}

Ключевые слова: симметрические функции, гипергеометрические функции, статистические суммы, тау-функции, матричные модели, решетки Тоды.

\section{1. РАЗБИЕНИЯ, ФУНКЦИИ ШУРА И СКАЛЯРНЫЕ ПРОИЗВЕДЕНИЯ}

1.1. Разбиения. Напомним стандартные определения и обозначения из теории симметрических функций и разбиений (см. более подробное изложение в книге [1]). Симметрические многочлены многих переменных параметризуются разбиениями. Разбиение представляет собой любую (конечную или бесконечную) последовательность неотрицательных целых чисел, записанную в порядке убывания:

$$
\lambda=\left(\lambda_{1}, \lambda_{2}, \ldots, \lambda_{r}, \ldots\right), \quad \lambda_{1} \geqslant \lambda_{2} \geqslant \cdots \geqslant \lambda_{r} \geqslant \cdots
$$

Ненулевые члены $\lambda_{i}$ в разбиении (1.1) называются членами разбиения $\lambda$; число членов равно длине разбиения $\lambda$ и обозначается как $l(\lambda)$, а сумма всех членов называется весом

${ }^{*}$ Centre de Recherches Mathématiques, Université de Montréal, C.P. 6128, succ. centre ville, Montréal, Québec, Canada H3C 3J7; Department of Mathematics and Statistics, Concordia University, 7141 Sherbrooke W., Montréal, Québec, Canada H4B 1R6. E-mail: harnad@crm.umontreal.ca

${ }^{\dagger}$ Институт океанологии РАН, Нахимовский пр. 36, 117851, Москва, Россия. E-mail: orlovs@wave.sio.rssi.ru 
разбиения $\lambda$ и обозначается через $|\lambda|$. Если $n=|\lambda|$, то будем говорить, что $\lambda$ является разбиением $n$. Разбиение нуля обозначается через (0).

Диаграмма разбиения (или диаграма Юнга) определяется как набор точек (или ячеек) $\left\{(i, j) \in \mathbb{Z}^{2}\right\}$ такой, что $1 \leqslant j \leqslant \lambda_{i}$. Поэтому диаграмму Юнга можно изобразить как подмножество матричных элементов в матрице с $l(\lambda)$ рядами и $\lambda_{1}$ столбцами. Диаграмма разбиения $\lambda$ обозначается тем же символом.

Например,

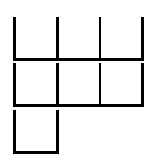

представляет собой диаграмму разбиения $(3,3,1)$. Вес этого разбиения равен 7 , а длина равна 3.

Другое определение разбиения восходит к Фробениусу. Предположим, что главная диагональ диаграммы $\lambda$ состоит из $r$ ячеек $(i, i), 1 \leqslant i \leqslant r$. Пусть $\alpha_{i}=\lambda_{i}-i$ - число ячеек в $i$-м ряду диаграммы $\lambda$ справа от $(i, i)$ при $1 \leqslant i \leqslant r$ и пусть $\beta_{i}=\lambda_{i}^{\prime}-i-$ число ячеек в $i$-м столбце диаграммы $\lambda$ ниже диагонали $(i, i)$ при $1 \leqslant i \leqslant r$. Имеем $\alpha_{1}>\alpha_{2}>\cdots>\alpha_{r} \geqslant 0$ и $\beta_{1}>\beta_{2}>\cdots>\beta_{r} \geqslant 0$. В обозначениях Фробениуса разбиение $\lambda$ обозначается как

$$
\lambda=\left(\alpha_{1}, \ldots, \alpha_{r} \mid \beta_{1}, \ldots, \beta_{r}\right)=(\alpha \mid \beta) .
$$

Это отвечает разложению диаграммы $\lambda$ по крюкам, в котором наибольший крюк имеет вид $\left(\alpha_{1} \mid \beta_{1}\right)$, следуюший за ним - $\left(\alpha_{2} \mid \beta_{2}\right)$ и т.д. вплоть до наименьшего крюка, который равен $\left(\alpha_{r} \mid \beta_{r}\right)$. Углы крюков расположены на главной диагонали диаграммы. Например, разбиение $(3,3,1)$ состоит из двух крюков $(2,2)$ и $(1,0)$ :

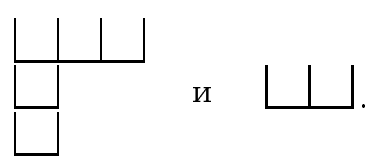

Таким образом, в обозначениях Фробениуса это разбиение будет иметь вид $(2,1 \mid 2,0)$.

1.2. Скалярные произведения функций Шура. В применении к теории уравнения Кадомцева-Петвиашвили (КП) полезно рассмотреть функции Шура $s_{\lambda}$ как базис в пространстве взвешенных однородных многочленов от бесконечного набора переменных $\gamma=\left(\gamma_{1}, \gamma_{2}, \ldots\right)$ (с весом $\left.\left(\gamma_{j}\right)=j\right)$, задаваемых формулами

$$
s_{\lambda}(\gamma)=\operatorname{det}\left(h_{\lambda_{i}-i+j}(\gamma)\right)_{1 \leqslant i, j \leqslant r},
$$

где $\left\{h_{n}(\gamma)\right\}_{n \in \mathbb{Z}}$ - элементарные функции Шура (или полностью симметрические функции), задаваемые разложением Тейлора вида

$$
e^{\xi(\boldsymbol{\gamma}, z)}:=\exp \left(\sum_{k=1}^{\infty} \gamma_{k} z^{k}\right)=\sum_{n=0}^{\infty} z^{n} h_{n}(\boldsymbol{\gamma}),
$$


а $h_{n}(\gamma) \equiv 0$ при $n<0$. Если задать параметры $\gamma=\left(\gamma_{1}, \gamma_{2}, \ldots\right)$ в терминах конечного набора переменных $\left(x_{1}, \ldots, x_{N}\right)$ формулой

$$
\gamma_{j}=\frac{1}{j} \sum_{a=1}^{N} x_{a}^{j}
$$

т.е. если функции Шура интерпретируются как неприводимые характеры представления $G L(N)$, то можно использовать обозначения $s_{\lambda}([\mathbf{x}])$, где

$$
[\mathbf{x}]:=\left(\sum_{a=1}^{N} x_{a}, \sum_{a=1}^{N} x_{a}^{2}, \ldots\right) .
$$

Тождество Коши-Литтлвуда [1] задает производящую функцию для полного набора функций Шура, рассматриваемых как функции от двух конечных наборов переменных $\left(x_{1}, \ldots, x_{N}\right),\left(y_{1}, \ldots, y_{M}\right)$ :

$$
\prod_{a=1}^{N} \prod_{b=1}^{M}\left(1-x_{a} y_{b}\right)^{-1}=\sum_{\lambda} s_{\lambda}(\mathbf{t}) s_{\lambda}\left(\mathbf{t}^{*}\right),
$$

где

$$
t_{j}=\frac{1}{j} \sum_{a=1}^{N} x_{a}^{j}, \quad t_{j}^{*}=\frac{1}{j} \sum_{a=1}^{M} y_{a}^{j}
$$

$\mathbf{t}:=\left(t_{1}, t_{2}, \ldots\right)$ и $\mathbf{t}^{*}:=\left(t_{1}^{*}, t_{2}^{*}, \ldots\right)$.

Скалярное произведение [1] задается на пространстве взвешенных однородных многочленов от бесконечной последовательности переменных, и оно таково, что функции Шура ортонормальны:

$$
\left\langle s_{\lambda}, s_{\mu}\right\rangle=\delta_{\mu, \lambda}
$$

В подходе иерархий КП и решетки Тоды (РТ) переменные $\left\{x_{i}\right\}$ в формуле (1.4) (возможно, при $N=\infty)$ называются переменными Хироты-Мивы, а набор $\gamma=\left(\gamma_{1}, \gamma_{2}, \ldots\right)$ параметризует поток иерархии КП.

\section{2. ДЕФОРМАЦИЯ СТАНДАРТНОГО СКАЛЯРНОГО ПРОИЗВЕДЕНИЯ И РЯДЫ ГИПЕРГЕОМЕТРИЧЕСКОГО ТИПА}

2.1. Скалярное произведение $\langle\cdot, \cdot\rangle_{r, n}$. Пусть даны функция $r(n)$ от целых чисел $n \in \mathbb{Z}$ и разбиение $\lambda$ веса $|\lambda|$ и длины $l(\lambda)$. Введем обозначение

$$
r_{\lambda}(n):=\prod_{i, j \in \lambda} r(n+j-i)=\prod_{i=1}^{l(\lambda)} \prod_{j=1}^{\lambda_{i}} r(n+j-i)
$$

Тем самым $r_{\lambda}(n)$ есть произведение значений функции $r$, сдвинутых на содержание ячейки $(j-i)$, взятое по всем ячейкам диаграммы Юнга разбиения $\lambda$. Например, для разбиения $(3,3,1)$ имеем

$$
r_{(3,3,1)}(n)=r(n+2)(r(n+1))^{2}(r(n))^{2} r(n-1) r(n-2) .
$$


Для нулевого разбиения положим $r_{0} \equiv 1$.

Для заданной функции $r$ и целого числа $n$, бо́льшего или равного максимальному нулю функции $r$, можно определить связанную с ними билинейную форму $\langle\cdot, \cdot\rangle_{r, n}$ :

$$
\left\langle s_{\lambda}, s_{\mu}\right\rangle_{r, n}=r_{\lambda}(n) \delta_{\lambda \mu}
$$

Пусть $\left\{n_{i} \in \mathbb{Z}\right\}$ - набор нулей функции $r$ и $k:=\min \left|n-n_{i}\right|$. Произведение (2.2) невырож денно на пространстве $\Lambda_{k}$ симметрических многочленов от $k$ переменных $\mathbf{x}^{k}:=$ $\left(x_{1}, \ldots, x_{k}\right)$. Это следует из того, что по определению (2.1) величина $r_{\lambda}(n)$ никогда не обрашается в нуль для разбиения $\lambda$ длины $l(\lambda) \leqslant k$. Тогда функции Шура от $k$ переменных $\left\{s_{\lambda}\left(\left[\mathrm{x}^{k}\right]\right), l(\lambda) \leqslant k\right\}$ образуют базис в пространстве $\Lambda_{k}$. Если, наоборот, $n-n_{i}<0$ для всех нулей функции $r$, то множитель $r_{\lambda}(n)$ никогда не обрашается в нуль для разбиения $\left\{\lambda: l\left(\lambda^{\prime}\right) \leqslant k\right\}$, где $\lambda^{\prime}-$ сопряженное разбиение, и $\left\{s_{\lambda}\left(-\left[\mathbf{x}^{k}\right]\right), l\left(\lambda^{\prime}\right) \leqslant k\right\}$ задают базис на $\Lambda_{k}$. Если функция $r$ не обрашается в нуль, то скалярное произведение невырожденно на $\Lambda_{\infty}$.

2.2. Основной пример. Случай $r(n)=n$ играет выделенную роль в приложениях к двухматричным моделям. Обозначим через $\langle\cdot, \cdot\rangle_{n}^{\prime}$ скалярное произведение в этом случае:

$$
\left\langle s_{\lambda}, s_{\mu}\right\rangle_{n}^{\prime}=(n)_{\lambda} \delta_{\lambda \mu}, \quad(n)_{\lambda}=\prod_{i=1}^{l(\lambda)} \prod_{j=1}^{\lambda_{i}}(n-i+j)
$$

(напомним, что ограничение его на любое пространство симметрических многочленов от $\leqslant n$ переменных невырожденно). Для пары $(f, g)$ симметрических функций от $n$ переменных $\mathbf{x}=\left(x_{1}, \ldots, x_{n}\right)$ получим следующую простую реализацию этого скалярного произведения:

$$
\langle f, g\rangle_{n}^{\prime}=\left.\frac{1}{c_{n}} \Delta(\partial) f(\partial) \cdot \Delta(\mathbf{x}) g(\mathbf{x})\right|_{\mathbf{x}=0}, \quad c_{n}=\prod_{k=1}^{n} k !
$$

где

$$
\Delta(\mathbf{x}):=\prod_{1 \leqslant i<j \leqslant n}\left(x_{i}-x_{j}\right)
$$

- определитель Вандермонда,

$$
\Delta(\partial):=\prod_{1 \leqslant i<j \leqslant n}\left(\frac{\partial}{\partial x_{i}}-\frac{\partial}{\partial x_{j}}\right)
$$

a $f(\partial)$ - оператор, получаемый при замене $\left\{x_{i}\right\}$ на $\left\{\partial / \partial x_{i}\right\}$. Доказательство соотношения (2.4) следует из формулы Якоби-Труди для функции Шура [1]

$$
s_{\lambda}(\mathbf{x})=\frac{\operatorname{det}\left(x_{i}^{\lambda_{j}+n-j}\right)_{i, j=1, \ldots, n}}{\Delta(\mathbf{x})}
$$


и формулы

$$
\left.\frac{1}{c_{n}} \operatorname{det}\left(\partial_{x_{i}}^{\lambda_{j}+n-j}\right)_{i, j=1, \ldots, n} \operatorname{det}\left(x_{i}{ }^{\mu_{j}+n-j}\right)_{i, j=1, \ldots, n}\right|_{\mathbf{x}=0}=(n)_{\lambda} \delta_{\lambda \mu}=\left\langle s_{\lambda}, s_{\mu}\right\rangle_{r, n} .
$$

(Интересно сравнить скалярное произведение (2.4) с произведением, построенным в работе [2].)

Представим две различные реализации формулы (2.4) с помощью следующих многократных интегралов.

A.

$$
\langle f, g\rangle_{n}^{\prime}=\frac{1}{\pi^{n} c_{n}} \int_{\mathbb{C}} \cdots \int_{\mathbb{C}} f(\mathbf{z}) g(\overline{\mathbf{z}}) e^{-\left|z_{1}\right|^{2}-\cdots-\left|z_{n}\right|^{2}}|\Delta(\mathbf{z})|^{2} d^{2} z_{1} \ldots d^{2} z_{n},
$$

где интегрирование идет по $n$ копиям $\left\{z_{i}\right\}_{i=1, \ldots, n}$ комплексной плоскости. Доказательство формулы (2.8) вытекает из следующей реализации, верной для любой пары полиномиальных функций $(a, b)$ от одной переменной:

$$
\left.a\left(\frac{\partial}{\partial z}\right) \cdot b(z)\right|_{z=0}=\frac{1}{\pi} \int_{C} a(z) b(\bar{z}) e^{-|z|^{2}} d z d \bar{z}
$$

Б.

$$
\begin{aligned}
& \langle f, g\rangle_{n}^{\prime}=\frac{1}{(2 \pi i)^{n} c_{n}} \times \\
& \quad \times \int_{\operatorname{Re}} \int_{\operatorname{Im}} \cdots \int_{\operatorname{Re}} \int_{\operatorname{Im}} f(\mathbf{x}) g(\mathbf{y}) e^{-\left(x_{1} y_{1}+\cdots+x_{n} y_{n}\right)} \Delta(\mathbf{x}) \Delta(\mathbf{y}) d x_{1} d y_{1} \ldots d x_{n} d y_{n},
\end{aligned}
$$

где интегрирование идет по $n$ копиям $\left\{x_{i}\right\}_{i=1, \ldots, n}$ вещественной прямой и $n$ копиям $\left\{y_{i}\right\}_{i=1, \ldots, n}$ мнимой прямой. Если $f$ и $g$ - многочлены, то эти интегралы могут быть вычислены в терминах обобшенных функций при подходяшей процедуре регуляризации. С другой стороны, можно интерпретировать реализацию (2.10) как примененную к паре функций $f, g$, которые убьвают достаточно быстро на бесконечности, чтобы обеспечить сходимость интеграла.

Доказательство реализации (2.10) следует из формулы

$$
\left.a\left(\frac{\partial}{\partial x}\right) \cdot b(x)\right|_{x=0}=\frac{1}{2 \pi i} \int_{\operatorname{Re}} \int_{\operatorname{Im}} a(x) b(y) e^{-x y} d x d y .
$$

Ниже в основном рассматриваются следуюшие ряды:

$$
\begin{gathered}
\left.\frac{1}{c_{n}} \exp \left(\sum_{m=1}^{\infty} \sum_{i=1}^{n} t_{m} \partial_{x_{i}}^{m}\right) \Delta(\partial) \cdot \exp \left(\sum_{m=1}^{\infty} \sum_{i=1}^{n} t_{m}^{*} x_{i}^{m}\right) \Delta(x)\right|_{\mathbf{x}=0}= \\
=\sum_{\lambda}(n)_{\lambda} s_{\lambda}(\mathbf{t}) s_{\lambda}\left(\mathbf{t}^{*}\right) .
\end{gathered}
$$

Это равенство можно получить из формул $(2.3),(2.4)$ и обобщенного тождества Коши-Литтлвуда (см. формулу (4.7) ниже). Правая часть, вообще говоря, расходится и должна рассматриваться как градуированный формальный полиномиальный ряд. Ее можно отождествить с гипергеометрическим рядом ${ }_{2} \mathcal{F}_{0}\left(n, n ; \mathbf{t}, \mathbf{t}^{*}\right)($ см. [3]). 


\section{3. ФЕРМИОННЫЕ ОПЕРАТОРЫ, ВАКУУМНЫЕ ОЖИДАНИЯ, ТАУ-ФУНКЦИИ}

3.1. Свободные фермионы. Воспользуемся формализмом фермионного пространства Фока, как было сделано в работе [4]. Алгебра свободных фермионов - это бесконечномерная алгебра Клиффорда $\mathbf{A}$ над полем $\mathbb{C}$ с генераторами $\psi_{n}, \psi_{n}^{*}, n \in \mathbb{Z}$, удовлетворяюшими соотношениям антикоммутации:

$$
\left[\psi_{m}, \psi_{n}\right]_{+}=\left[\psi_{m}^{*}, \psi_{n}^{*}\right]_{+}=0, \quad\left[\psi_{m}, \psi_{n}^{*}\right]_{+}=\delta_{m n}, \quad m, n \in \mathbb{Z}
$$

Любой элемент пространства $W=\left(\bigoplus_{m \in \mathbb{Z}} \mathbb{C} \psi_{m}\right) \oplus\left(\bigoplus_{m \in \mathbb{Z}} \mathbb{C} \psi_{m}^{*}\right)$ называется свободным фермионом. Алгебра Клиффорда допускает следуюшее стандартное представление (представление Фока). Пусть $W_{\text {an }}=\left(\bigoplus_{m<0} \mathbb{C} \psi_{m}\right) \oplus\left(\bigoplus_{m \geqslant 0} \mathbb{C} \psi_{m}^{*}\right)$ и $W_{\mathrm{cr}}=$ $\left(\bigoplus_{m \geqslant 0} \mathbb{C} \psi_{m}\right) \oplus\left(\bigoplus_{m<0} \mathbb{C} \psi_{m}^{*}\right)$. Рассмотрим левый и правый $\mathbf{A}$-модули $F=\mathbf{A} / \mathbf{A} W_{\text {an }}$ и $F^{*}=W_{\mathrm{cr}} \mathbf{A} \backslash \mathbf{A}$, соответственно. Они представляют собой циклические $\mathbf{A}$-модули, порожденные векторами $|0\rangle=1\left(\bmod \mathbf{A} W_{\text {an }}\right)$ и $\langle 0|=1\left(\bmod W_{\text {cr }} \mathbf{A}\right)$, соответственно, со свойствами

$$
\begin{aligned}
& \psi_{m}|0\rangle=0 \quad(m<0), \quad \psi_{m}^{*}|0\rangle=0 \quad(m \geqslant 0), \\
& \langle 0| \psi_{m}=0 \quad(m \geqslant 0), \quad\langle 0| \psi_{m}^{*}=0 \quad(m<0) .
\end{aligned}
$$

Векторы $\langle 0|$ и $|0\rangle$ называются левым и правым вакуумными векторами. Фермионы $w \in$ $W_{\text {an }}$ уничтожают левый вакуум, в то время как фермионы $w \in W_{\text {cr } у \text { уничтожают правый }}$ вакуум.

Пространства Фока $F$ и $F^{*}$ взаимно дуальны, спаривание между ними задается линейной формой $\langle 0|\cdot| 0\rangle$ на А и называется вакуумным ожсданием. Оно дается формулами

$$
\begin{gathered}
\langle 0|1| 0\rangle=1, \quad\left\langle 0\left|\psi_{m} \psi_{m}^{*}\right| 0\right\rangle=1 \quad(m<0), \quad\left\langle 0\left|\psi_{m}^{*} \psi_{m}\right| 0\right\rangle=1 \quad(m \geqslant 0), \\
\left\langle 0\left|\psi_{m} \psi_{n}\right| 0\right\rangle=\left\langle 0\left|\psi_{m}^{*} \psi_{n}^{*}\right| 0\right\rangle=0, \quad\left\langle 0\left|\psi_{m} \psi_{n}^{*}\right| 0\right\rangle=0 \quad(m \neq n)
\end{gathered}
$$

и правилом Вика, имеющим вид

$$
\begin{gathered}
\left\langle 0\left|w_{1} \ldots w_{2 n+1}\right| 0\right\rangle=0 \\
\left\langle 0\left|w_{1} \ldots w_{2 n}\right| 0\right\rangle=\sum_{\sigma} \operatorname{sgn} \sigma\left\langle 0\left|w_{\sigma(1)} w_{\sigma(2)}\right| 0\right\rangle \ldots\left\langle 0\left|w_{\sigma(2 n-1)} w_{\sigma(2 n)}\right| 0\right\rangle,
\end{gathered}
$$

где $w_{k} \in W$ и $\sigma$ перечисляет перестановки такие, что $\sigma(1)<\sigma(2), \sigma(3)<\sigma(4), \ldots$, $\sigma(2 n-1)<\sigma(2 n)$ и $\sigma(1)<\sigma(3)<\cdots<\sigma(2 n-1), \sigma(k)$ есть образ числа $k$ при перестановке $\sigma$. 


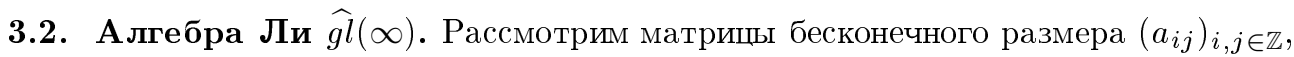
которые удовлетворяют следуюшему условию: сушествует $N$ такое, что $a_{i j}=0$ при $|i-j|>N$. Такие матрицы называются обобшенными матрицами Якоби (или “ленточными" матрицами), и они образуют алгебру Ли относительно обычной скобки матричного коммутатора.

Обозначим через : . . . : оператор нормального упорядочения, определенный таким образом, что

$$
: \psi_{i} \psi_{j}^{*}:=\psi_{i} \psi_{j}^{*}-\left\langle 0\left|\psi_{i} \psi_{j}^{*}\right| 0\right\rangle
$$

Линейные комбинации квадратичных элементов вида

$$
\sum_{i, j \in \mathbb{Z}} a_{i j}: \psi_{i} \psi_{j}^{*}
$$

вместе с единичным элементом образуют бесконечномерную алгебру Ли $\widehat{g l}(\infty)$ :

$$
\left[\sum_{i, j \in \mathbb{Z}} a_{i j}: \psi_{i} \psi_{j}^{*}:, \sum_{i, j \in \mathbb{Z}} b_{i j}: \psi_{i} \psi_{j}^{*}:\right]=\sum_{i, j \in \mathbb{Z}} c_{i j}: \psi_{i} \psi_{j}^{*}:+c_{0},
$$

где

$$
c_{i j}=\sum_{k} a_{i k} b_{k j}-\sum_{k} b_{i k} a_{k j}
$$

Последнее слагаемое в равенстве (3.4),

$$
c_{0}=\sum_{i<0, j \geqslant 0} a_{i j} b_{j i}-\sum_{i \geqslant 0, j<0} a_{i j} b_{j i},
$$

представляет собой центральный элемент, так что алгебра Ли $\widehat{g l}(\infty)$ есть центральное расширение алгебры обобщенных матрищ Якоби.

3.3. Билинейное тождество. Пусть $g$-экспонента оператора в $\widehat{g l}(\infty)$ :

$$
g=\exp \left(\sum_{i, j \in \mathbb{Z}} a_{i j}: \psi_{i} \psi_{j}:\right)
$$

т.е. является элементом соответствуюшей группы. Используя соотношение (3.4), можно вывести следующие соотношения:

$$
g \psi_{n}=\sum_{m} \psi_{m} A_{m n} g, \quad \psi_{n}^{*} g=g \sum_{m} A_{n m} \psi_{m}^{*}
$$

где коэффициенты $A_{n m}$ задаются числами $a_{n m}$. В свою очередь, из соотношений $(3.5)$ следует, что [4]

$$
\left[\sum_{n \in \mathbb{Z}} \psi_{n} \otimes \psi_{n}^{*}, g \otimes g\right]=0 .
$$

Это последнее соотношение очень важно с точки зрения приложений к интегрируемым системам, и из него следуют билинейные тождества Хироты. 
3.4. Параметры двойных потоков КП. Тау-функции КП и РТ. Введем следуюшие (гамильтоновы) операторы:

$$
H_{n}=\sum_{k=-\infty}^{+\infty} \psi_{k} \psi_{k+n}^{*}, \quad n \neq 0, \quad H(\mathbf{t})=\sum_{n=1}^{+\infty} t_{n} H_{n}, \quad H^{*}\left(\mathbf{t}^{*}\right)=\sum_{n=1}^{+\infty} t_{n}^{*} H_{-n} .
$$

Здесь функции $H_{n} \in \widehat{g l}(\infty)$ и $H(\mathbf{t}), H^{*}\left(\mathbf{t}^{*}\right)$ также лежат в алгебре $\widehat{g l}(\infty)$, если считать число ненулевых параметров $\left\{t_{m}, t_{m}^{*}\right\}$ конечным. Операторы $H_{n}$ удовлетворяют алгебре коммутационных соотношений Гейзенберга:

$$
\left[H_{n}, H_{m}\right]=n \delta_{m+n, 0}
$$

Заметим также, что

$$
H_{n}|0\rangle=0, \quad\langle 0| H_{-n}=0, \quad n>0 .
$$

В дальнейшем нам понадобятся также операторы свободного фермионного поля

$$
\psi(z):=\sum_{k} \psi_{k} z^{k}, \quad \psi^{*}(z):=\sum_{k} \psi_{k}^{*} z^{-k-1} d z
$$

(оператор $\psi^{*}$ здесь записан как 1-форма).

Для каждого $n \in \mathbb{N}^{+}$зададим вакуумные векторы “заряда $n$ ", пронумерованные целым числом $n$ :

$$
\begin{gathered}
\left\langle n\left|:=\left\langle 0\left|\Psi_{n}^{*}, \quad\right| n\right\rangle:=\Psi_{n}\right| 0\right\rangle, \\
\Psi_{n}= \begin{cases}\psi_{n-1} \ldots \psi_{1} \psi_{0}, & n>0, \\
\psi_{n}^{*} \ldots \psi_{-2}^{*} \psi_{-1}^{*}, & n<0,\end{cases} \\
\Psi_{n}^{*}= \begin{cases}\psi_{0}^{*} \psi_{1}^{*} \ldots \psi_{n-1}^{*}, & n>0, \\
\psi_{-1} \psi_{-2} \ldots \psi_{n}, & n<0 .\end{cases}
\end{gathered}
$$

Для любого $g$, удовлетворяюшего билинейному тождеству (3.6), соответствуюшие тау-функции иерархий КП и двумеризованной РТ имеют вид [4]

$$
\begin{aligned}
\tau_{\mathrm{K} \Pi}(n, \mathbf{t}) & :=\left\langle n\left|e^{H(\mathbf{t})} g\right| n\right\rangle, \\
\tau_{\mathrm{PT}}\left(n, \mathbf{t}, \mathbf{t}^{*}\right) & :=\left\langle n\left|e^{H(\mathbf{t})} g e^{H^{*}\left(\mathbf{t}^{*}\right)}\right| n\right\rangle .
\end{aligned}
$$

Первый набор переменных, появляюшихся в $\tau_{\mathrm{K \Pi}}(n, \mathbf{t}),-$ это обычные высшие времена уравнения $\mathrm{K}$, в то время как параметры $\mathbf{t}=\left(t_{1}, t_{2}, \ldots\right)$ и $\mathbf{t}^{*}=\left(t_{1}^{*}, t_{2}^{*}, \ldots\right)$ в $\tau_{\text {Рт }}\left(n, \mathbf{t}, \mathbf{t}^{*}\right)$ иногда называют двойным набором высших тодовских времен [4], [5]. (Если зафиксировать $n$ и параметры $\mathbf{t}^{*}$, то $\left\{\tau_{\mathrm{PT}}\left(n, \mathbf{t}, \mathbf{t}^{*}\right)\right\}_{n \in \mathbb{N}}$ можно также рассматривать как тау-функции иерархии КП от $\mathbf{t}$ переменных.) Первые три параметра $\left(t_{1}, t_{2}, t_{3}\right)$ суть независимые переменные, возникаюшие в собственно уравнении КП [6]:

$$
4 \partial_{t_{1}} \partial_{t_{3}} u=\partial_{t_{1}}^{4} u+3 \partial_{t_{2}}^{2} u+3 \partial_{t_{1}}^{2} u^{2}, \quad u=2 \partial_{t_{1}}^{2} \ln \tau .
$$




\section{4. ТАУ-ФУНКЦИЯ КП $\tau_{r}\left(n, \mathbf{t}, \mathbf{t}^{*}\right)$}

4.1. Разложение по функциям Шура. При каждом выборе параметров $\mathbf{t}^{*}=$ $\left(t_{1}^{*}, t_{2}^{*}, \ldots\right)$ определим оператор

$$
A\left(\mathbf{t}^{*}\right):=\sum_{m=1}^{\infty} t_{k}^{*} A_{k}
$$

где элементы $A_{k}$, имеющие вид

$$
A_{k}=\sum_{n=-\infty}^{+\infty} \psi_{n-k}^{*} \psi_{n} r(n) r(n-1) \ldots r(n-k+1), \quad k=1,2, \ldots,
$$

взаимно коммутируют:

$$
\left[A_{k}, A_{j}\right]=0 \quad \forall j, k .
$$

С помощью явного вида $A_{k}$ и соотношений антикоммутации (3.1) получим

$$
\begin{aligned}
e^{A\left(\mathbf{t}^{*}\right)} \psi(z) e^{-A\left(\mathbf{t}^{*}\right)} & =e^{-\xi_{r}\left(\mathbf{t}^{*}, z^{-1}\right)} \cdot \psi(z), \\
e^{A\left(\mathbf{t}^{*}\right)} \psi^{*}(z) e^{-A\left(\mathbf{t}^{*}\right)} & =e^{\xi_{r^{\prime}}\left(\mathbf{t}^{*}, z^{-1}\right)} \cdot \psi^{*}(z),
\end{aligned}
$$

где $\xi_{r}\left(\mathbf{t}^{*}, z^{-1}\right)$ и $\xi_{r^{\prime}}\left(\mathbf{t}^{*}, z^{-1}\right)$ - операторы на функциях от вспомогательного параметра $z$, имеющие вид

$$
\begin{gathered}
\xi_{r}\left(\mathbf{t}^{*}, z^{-1}\right):=\sum_{m=1}^{+\infty} t_{m}\left(\frac{1}{z} r(D)\right)^{m}, \quad \xi_{r^{\prime}}\left(\mathbf{t}^{*}, z^{-1}\right):=\sum_{m=1}^{+\infty} t_{m}\left(\frac{1}{z} r^{\prime}(D)\right)^{m} \\
D:=z \frac{d}{d z}, \quad r^{\prime}(D):=r(-D) .
\end{gathered}
$$

Операторы $r(D), r^{\prime}(D)$ действуют на базисе функций на окружности в соответствии с правилом

$$
r(D) \cdot z^{n}=r(n) z^{n}, \quad r^{\prime}(D) \cdot z^{n}=r(-n) z^{n} .
$$

Экспоненты в выражениях (4.3) задаются своими разложениями Тейлора.

Используя соотношения (4.3) и то, что под знаком $\operatorname{res}_{z}$ оператор $z^{-1} r(D)$ сопряжен оператору $z^{-1} r(-D)=z^{-1} r^{\prime}(D)$, получим следующую лемму.

ЛЕмма 1. Фермионный оператор $e^{A\left(\mathbf{t}^{*}\right)}$ удовлетворяет билинейному тожсдеству (3.6):

$$
\left[\operatorname{res}_{z=0} \psi(z) \otimes \psi^{*}(z), e^{A\left(\mathbf{t}^{*}\right)} \otimes e^{A\left(\mathbf{t}^{*}\right)}\right]=0 .
$$

Доказательство этой леммы следует из общего рассмотрения в работе [4].

С учетом формулы (4.4) выражение

$$
\tau_{r}\left(n, \mathbf{t}, \mathbf{t}^{*}\right):=\left\langle n\left|e^{H(\mathbf{t})} e^{-A\left(\mathbf{t}^{*}\right)}\right| n\right\rangle
$$

задает при каждом выборе параметров $n, r$ и $\mathbf{t}^{*}$ тау-функцию КП (3.11). При любом выборе $r$ функция $\tau_{r}\left(n, \mathbf{t}, \mathbf{t}^{*}\right)$, рассматриваемая как функция от всех переменных, оказывается тау-функцией двумеризованной РТ. 
ПРЕДЛОЖЕнИЕ 1. Справедливо равенство

$$
\tau_{r}\left(n, \mathbf{t}, \mathbf{t}^{*}\right)=\sum_{\lambda} r_{\lambda}(n) s_{\lambda}(\mathbf{t}) s_{\lambda}\left(\mathbf{t}^{*}\right),
$$

где операторнозначная әкспоненциальная функция $e^{-A\left(\mathbf{t}^{*}\right)}$ в обкладках вакуумного ожидания задается своим рядом Тейлора, а правая часть соотношения рассматривается как формальный ряд (сумма в формуле (4.6) должна включать разбиение нуля).

Заметим, что тау-функция (4.6) может рассматриваться как функция, получаемая при действии дополнительных симметрий [7], [8] на вакуумную тау-функцию. Переменные $\mathbf{t}$ играют роль параметров потока КП, и $\mathbf{t}^{*}$ при этом является набором групповых параметров, задающих показатель экспоненты, выражающий действие подалгебры дополнительных симметрий иерархии КП (см. [8]). С другой стороны, выражение (4.6) может рассматриваться как тау-функция двумеризованной РТ [5], [9] с двумя наборами непрерывных переменных $\mathbf{t}, \mathbf{t}^{*}$ и одной дискретной переменной $n$. Формула (4.6), очевидно, симметрична относительно перестановки $\mathbf{t} \leftrightarrow \mathbf{t}^{*}$.

4.2. Обобщенное тождество Коши-Литтлвуда и доказательство предложения 1. Мы будем понимать под обобшенным тождеством Коши-Литтлвуда следуюшее соотношение, которое может пониматься как (двойная) производящая функция для функций Шура:

$$
\exp \left(\sum_{m=1}^{\infty} m t_{m} t_{m}^{*}\right)=\sum_{\lambda} s_{\lambda}(\mathbf{t}) s_{\lambda}\left(\mathbf{t}^{*}\right) .
$$

В случае, когда параметры $\mathbf{t}=\left(t_{1}, t_{2}, \ldots\right)$ и $\mathbf{t}^{*}=\left(t_{1}^{*}, t_{2}^{*}, \ldots\right)$ задаются разложениями (1.6) в терминах двух конечных наборов переменных $\left(x_{1}, \ldots, x_{N}\right)$ и $\left(y_{1}, \ldots, y_{M}\right)$, это тождество сводится к тождеству Коши-Литтлвуда в обычной форме записи (1.5). Это можно доказать с использованием фермионного пространства Фока и формул скалярного произведения, которые обсуждаются ниже (в более ранних работах [3], [10] были представлены лишь наброски требуемых доказательств).

Для доказательства нам потребуется

Лемма 2 [4]. В случае, когда $-j_{1}<\cdots<-j_{k}<0 \leqslant i_{k}<\cdots<i_{1}$, имеет место следующая форммлл:

$$
\begin{aligned}
& \left\langle 0\left|e^{H(\mathbf{t})} \psi_{-j_{1}}^{*} \ldots \psi_{-j_{k}}^{*} \psi_{i_{k}} \ldots \psi_{i_{1}}\right| 0\right\rangle= \\
& \quad=\left\langle n\left|e^{H(\mathbf{t})} \psi_{-j_{1}+n}^{*} \ldots \psi_{-j_{k}+n}^{*} \psi_{i_{k}+n} \ldots \psi_{i_{1}+n}\right| n\right\rangle=(-1)^{j_{1}+\cdots+j_{k}} s_{\lambda}(\mathbf{t}),
\end{aligned}
$$

где разбиение $\lambda=\left(\lambda_{1}, \ldots, \lambda_{j_{1}}\right)$ задается условием

$$
\left(\lambda_{1}, \ldots, \lambda_{j_{1}}\right)=\left(i_{1}, \ldots, i_{k} \mid j_{1}-1, \ldots, j_{k}-1\right),
$$

әде $(\ldots \mid \ldots)$ - разбиение в обозначениях Фробениуса.

Доказательство этой леммы состоит в прямом вычислении с использованием правила Вика [4]. 
Введем теперь векторы (ср. с работой [11])

$$
\begin{aligned}
& |\lambda, n\rangle:=\psi_{-j_{1}+n}^{*} \ldots \psi_{-j_{k}+n}^{*} \psi_{i_{k}+n} \ldots \psi_{i_{1}+n}|n\rangle \\
& \langle\lambda, n|:=\langle n| \psi_{i_{1}+n}^{*} \ldots \psi_{i_{k}+n}^{*} \psi_{-j_{k}+n} \ldots \psi_{-j_{1}+n}
\end{aligned}
$$

Получим

$$
\langle\lambda, n \mid \mu, m\rangle=\delta_{m n} \delta_{\lambda \mu},
$$

так что наборы векторов (4.10) и (4.11) задают ортонормированные базисы в пространстве Фока $F$ и дуальном к нему пространстве $F^{*}$, соответственно.

Для всякого разбиения $\lambda$, выраженного в фробениусовом виде (4.9), зададим целое число

$$
a(\lambda):=j_{1}+\cdots+j_{k}
$$

Тогда из леммы 2 и соотношений ортогональности (4.12) следуют разложения

$$
\langle n| e^{H(\mathbf{t})}=\sum_{\lambda}(-1)^{a(\lambda)} s_{\lambda}(\mathbf{t})\langle\lambda, n|
$$

и

$$
e^{H^{*}\left(\mathbf{t}^{*}\right)}|n\rangle=\sum_{\lambda}|\lambda, n\rangle(-1)^{a(\lambda)} s_{\lambda}\left(\mathbf{t}^{*}\right)
$$

ДОКАЗАТЕЛЬСТВО ОБОБШЕННОГО ТОЖДЕСТВА КОШИ-ЛИТТЛВУДА. Рассмотрим вакуумную тау-функцию РТ

$$
\left\langle 0\left|e^{H(\mathbf{t})} e^{H^{*}\left(\mathbf{t}^{*}\right)}\right| 0\right\rangle
$$

Из формул (4.13), (4.14) получаем, что она равна правой части соотношения (4.7):

$$
\left\langle 0\left|e^{H(\mathbf{t})} e^{H^{*}\left(\mathbf{t}^{*}\right)}\right| 0\right\rangle=\sum_{\lambda} s_{\lambda}(\mathbf{t}) s_{\lambda}\left(\mathbf{t}^{*}\right),
$$

где в сумму опять входит разбиение нуля.

Из коммутационных соотношений алгебры Гейзенберга (3.8) следует, что сопряжение элемента $e^{H_{-m}}$ элементом $e^{H_{m}}$ сводится к простому сдвигу показателя экспоненты на $m$. Это вместе с уравнениями (3.9) приводит к тому, что вакуумная тау-функция PТ (4.15) оказывается равной левой части уравнения (4.7):

$$
\left\langle 0\left|e^{H(\mathbf{t})} e^{H^{*}\left(\mathbf{t}^{*}\right)}\right| 0\right\rangle=\exp \left(\sum_{m=1}^{\infty} m t_{m} t_{m}^{*}\right) .
$$

3 Теоретическая и математическая физика, т. 137, № 3, 2003 г. 
ДОКАЗАТЕЛЬСТВО ПРЕДЛОЖЕНИЯ 1. Рассмотрим разложение в ряд Тейлора по всем переменным времен $t_{1}, t_{2}, \ldots$, задаваемое формулой

$$
e^{A\left(\mathbf{t}^{*}\right)}|n\rangle=\sum_{n_{1}, n_{2}, \ldots=0}^{\infty} t_{1}^{n_{1}} t_{2}^{n_{2}} \ldots A_{1}^{n_{1}} A_{2}^{n_{2}} \ldots|n\rangle .
$$

Поскольку все величины $A_{k}$ взаимно коммутируют, порядок сомножителей в правой части несуществен. Каждая величина $A_{k}$ имеет следуюшую структуру:

$$
A_{k}=\sum_{m=-\infty}^{+\infty} e_{m, m-k}
$$

где

$$
e_{m, m-i}:=\psi_{m} \psi_{m-i}^{*} r(m) r(m-1) \ldots r(m-i+1) .
$$

Напомним, что

$$
\psi_{m}|n\rangle=0, \quad m<n, \quad \psi_{m}^{*}|n\rangle=0, \quad m \geqslant n .
$$

При заданном $n$ удобно разложить $\widehat{g l}(\infty)$ (как линейное пространство, а не как алгебру Ли) в следующую прямую сумму:

$$
a=a^{++} \oplus a^{--} \oplus a^{+-} \oplus a^{-+}, \quad a \in \widehat{g l}(\infty),
$$

где

$$
\begin{array}{ll}
a^{++}=\sum_{i, k \geqslant n}: \psi_{i} \psi_{k}^{*}: a_{i k}, & a^{--}=\sum_{i, k<n}: \psi_{i} \psi_{k}^{*}: a_{i k}, \\
a^{+-}=\sum_{i \geqslant n, k<n}: \psi_{i} \psi_{k}^{*}: a_{i k}, & a^{-+}=\sum_{i<n, k \geqslant n}: \psi_{i} \psi_{k}^{*}: a_{i k} .
\end{array}
$$

Далее получим

$$
\left[e_{i, j}^{+-}, e_{k, m}^{+-}\right]=0, \quad\left[A_{i}^{+-}, A_{k}^{+-}\right]=0,
$$

в то время как

$$
\left[e_{i, m}^{++}, e_{m, k}^{+-}\right]=e_{i, k}^{+-}, \quad\left[e_{i, m}^{+-}, e_{m, k}^{--}\right]=e_{i, k}^{+-} .
$$

Каждый член в сумме в правой части (4.16) представляет собой линейную комбинацию мономов вида

$$
e_{i_{1}, k_{1}} \ldots e_{i_{N}, k_{N}}|n\rangle
$$

при некотором $N$. Произведение содержит члены $e_{i, k}^{+-}, e_{i, k}^{++}, e_{i, k}^{--}$(с различными нижними индексами $i, k)$, из которых два последних члена уничтожают вакуумный вектор $|n\rangle$. С помошью коммутационных соотношений (4.18) можно пронести все элементы 
$e_{i, k}^{++}, e_{i, k}^{--}$направо в формуле (4.19), после чего это выражение превратится в произведение, содержашее только члены $e_{i, k}^{+-}$:

$$
e_{i_{1}, k_{1}}^{+-} \ldots e_{i_{M}, k_{M}}^{+-}|n\rangle, \quad M \leqslant N
$$

Рассмотрим теперь выражение (при $i>0$ )

$$
H_{-i}=\sum_{m=-\infty}^{+\infty} E_{m, m-i}
$$

где

$$
E_{m, m-i}:=\psi_{m} \psi_{m-i}^{*}
$$

в терминах которого можно записать

$$
\begin{gathered}
e_{m, m-i}:=E_{m, m-i} r(m) r(m-1) \ldots r(m-i+1), \\
e_{i_{1}, k_{1}}^{+-} \ldots e_{i_{M}, k_{M}}^{+-}|n\rangle=r_{\lambda}(n) E_{i_{1}, k_{1}}^{+-} \ldots E_{i_{M}, k_{M}}^{+-}|n\rangle .
\end{gathered}
$$

Заметим, что в выражении $E_{i_{1}, k_{1}}^{+-} \ldots E_{i_{M}, k_{M}}^{+-}|n\rangle$ вьполняется $E_{i, k}^{+-} E_{j, m}^{+-}=E_{i, m}^{+-} E_{j, k}^{+-}$, а потому с помощью действия группы перестановок и перестановочных соотношений (4.17) можно переставить все первые индексы в убываюшем порядке, т.е. $i_{1} \geqslant i_{2} \geqslant \cdots$, а для вторых индексов добиться того, чтобы было выполнено $-k_{1} \geqslant-k_{2} \geqslant \cdots$. Тогда получим

$$
e_{i_{1}, k_{1}}^{+-} \ldots e_{i_{M}, k_{M}}^{+-}|n\rangle=r_{\lambda}(n) E_{i_{1}, k_{1}}^{+-} \ldots E_{i_{M}, k_{M}}^{+-}|n\rangle=r_{\lambda}(n)|\lambda, n\rangle(-1)^{M},
$$

где разбиение $\lambda$ имеет следуюший вид в обозначениях Фробениуса:

$$
\lambda=\left(i_{1}, \ldots, i_{M} \mid-k_{1}-1, \ldots,-k_{M}-1\right) .
$$

Поскольку из (4.14) следует, что

$$
e^{H^{*}\left(\mathbf{t}^{*}\right)}|n\rangle=\sum_{\lambda}|\lambda, n\rangle(-1)^{a(\lambda)} s_{\lambda}\left(\mathbf{t}^{*}\right),
$$

то окончательно получим

$$
e^{A\left(\mathbf{t}^{*}\right)}|n\rangle=\sum_{\lambda}|\lambda, n\rangle(-1)^{a(\lambda)} r_{\lambda}(n) s_{\lambda}\left(\mathbf{t}^{*}\right),
$$

что и доказывает представление (4.6). Отсюда также вытекает следующий результат. 
ПРЕДЛОЖЕнИЕ 2 [10]. Справедливо равенство

$$
\left\langle s_{\lambda}, s_{\mu}\right\rangle_{r, n}=\left\langle n\left|s_{\lambda}(\mathbf{H}) s_{\mu}(-\mathbf{A})\right| n\right\rangle
$$

где переменные $\left(\gamma_{1}, \gamma_{2}, \ldots\right)$ в аргументе функций Шура (см. (1.2)) заменяются на операторы

$$
\mathbf{H}:=\left(\frac{H_{1}}{1}, \frac{H_{2}}{2}, \frac{H_{3}}{3}, \ldots\right), \quad-\mathbf{A}:=\left(-\frac{A_{1}}{1},-\frac{A_{2}}{2},-\frac{A_{3}}{3}, \ldots\right) .
$$

ДокАЗАТЕЛЬСТво. Из обобшенного тождества Коши-Литтлвуда следует, что

$$
\left\langle n\left|e^{H(\mathbf{t})}=\sum_{\lambda} s_{\lambda}(\mathbf{t})\left\langle n\left|s_{\lambda}(\mathbf{H}), \quad e^{A\left(\mathbf{t}^{*}\right)}\right| n\right\rangle=\sum_{\mu} s_{\mu}(-\mathbf{A})\right| n\right\rangle s_{\mu}\left(\mathbf{t}^{*}\right) .
$$

Из выражений (4.21), (4.13) и (4.12) следует, что формула (4.23) эквивалентна представлению (4.24) из нижеследуюшего предложения, с помошью которого можно выразить тау-функцию $\tau_{r}\left(n, \mathbf{t}, \mathbf{t}^{*}\right)$ в терминах скалярного произведения $\langle\cdot, \cdot\rangle_{r, n}$.

ПРеДЛОЖЕНИЕ 3 [10]. Справедливо равенство

$$
\tau_{r}\left(n, \mathbf{t}, \mathbf{t}^{*}\right)=\left\langle\exp \left(\sum_{m=1}^{\infty} m t_{m} \gamma_{m}\right), \exp \left(\sum_{m=1}^{\infty} m t_{m}^{*} \gamma_{m}\right)\right\rangle_{r, n},
$$

где рассматривается скалярное произведение на пространстве функиий от переменных $\gamma$, в то время как величины $\mathbf{t}, \mathbf{t}^{*}$ рассматриваются как параметры.

\section{5. МАТРИЧНЫЕ МОДЕЛИ}

5.1. Модели нормальных матриц. Рассмотрим ансамбль нормальных матриц $M$ размера $n \times n$ (т.е. матриц, коммутируюших со своими эрмитово сопряженными). Статистическая сумма модели нормальных матриц задается интегралом [12]

$$
\begin{aligned}
& I^{N M}\left(n, \mathbf{t}, \mathbf{t}^{*} ; \mathbf{u}\right)= \\
& \quad=C_{n} \int_{\mathbb{C}} \cdots \int_{\mathbb{C}}|\Delta(\mathbf{z})|^{2} \exp \left(\sum_{i=1}^{n}\left(V_{1}\left(z_{i}\right)+V_{2}\left(\bar{z}_{i}\right)-z_{i} \bar{z}_{i}\right)\right) \prod_{i=1}^{n} d z_{i} d \bar{z}_{i} .
\end{aligned}
$$

(Эта модель имеет приложения, например в задаче с лапласовым ростом [13], описываюшей динамику гранишы нефтяной пленки на поверхности воды.) Последний интеграл в формуле (5.1) берется по $n$ копиям комплексной плоскости, $\left(z_{1}, \ldots, z_{n}\right)$ - собственные значения матрицы $M$, а $C_{n}$ - нормировочный множитель, возникающий при интегрировании по угловым матричным переменным, $V_{1}$ и $V_{2}$ - два ряда следуюшего вида:

$$
V_{1}(z):=\sum_{m=1}^{\infty} t_{m} z^{m}, \quad V_{2}(z):=\sum_{m=1}^{\infty} t_{m}^{*} z^{m}
$$


Формула (2.8) при этом задает разложение в ряд для интеграла (5.1):

$$
\begin{aligned}
I^{N M}\left(n, \mathbf{t}, \mathbf{t}^{*} ; \mathbf{u}\right) & =\left\langle\exp \left(\sum_{i=1}^{n} \sum_{m=1}^{\infty} t_{m} z_{i}^{m}\right), \exp \left(\sum_{i=1}^{n} \sum_{m=1}^{\infty} t_{m}^{*} z_{i}^{m}\right)\right\rangle_{n}^{\prime}= \\
& =\sum_{\lambda}(n)_{\lambda} s_{\lambda}(\mathbf{t}) s_{\lambda}\left(\mathbf{t}^{*}\right) .
\end{aligned}
$$

Эту формулу можно интерпретировать [14], [15] как многомерный аналог суммирования по Борелю для ряда в правой части, которьй, вообще говоря, расходится.

5.2. Двухматричная модель. Вычислим теперь следуюшую статистическую сумму, заданную на ансамбле из пар $\left(M_{1}, M_{2}\right)$, где $M_{1}$ - эрмитова матрица размера $n \times n$, а $M_{2}$ - антиэрмитова матрица размера $n \times n$ :

$$
I^{2 M M}\left(n, \mathbf{t}, \mathbf{t}^{*}\right)=\int e^{\operatorname{tr}\left(V_{1}\left(M_{1}\right)+V_{2}\left(M_{2}\right)-M_{1} M_{2}\right)} d M_{1} d M_{2} .
$$

Хорошо известно [16], [17], что этот интеграл сводится к следуюшему интегралу по собственным значениям $x_{i}$ и $y_{i}$ соответствуюших матриц $M_{1}$ и $M_{2}$ :

$$
\widetilde{C}_{n} \int_{\operatorname{Re}} \int_{\operatorname{Im}} \cdots \int_{\operatorname{Re}} \int_{\operatorname{Im}} \exp \left(\sum_{j=1}^{n}\left(\sum_{m=1}^{\infty}\left(t_{m} x_{j}^{m}+t_{m}^{*} y_{j}^{m}\right)-x_{j} y_{j}\right)\right) \Delta(\mathbf{x}) \Delta(\mathbf{y}) \prod_{j=1}^{n} d x_{j} d y_{j},
$$

где нормировочный множитель $\widetilde{C}_{n}$ пропорционален объему унитарной группы. В работе [18] было показано, что этот интеграл задает тау-функцию двумеризованной РТ.

Из представления (2.10) следует, что статистическая сумма в такой модели случайных матриц представляется рядом гипергеометрического типа:

$$
\begin{aligned}
I^{2 M M}\left(n, \mathbf{t}, \mathbf{t}^{*}\right) & =\frac{\widetilde{C}_{n}}{(2 \pi i)^{n}}\left\langle\exp \left(\sum_{i=1}^{n} \sum_{m=1}^{\infty} t_{m} z_{i}^{m}\right), \exp \left(\sum_{i=1}^{n} \sum_{m=1}^{\infty} t_{m}^{*} z_{i}^{m}\right)\right\rangle_{n}^{\prime}= \\
& =\frac{\widetilde{C}_{n}}{(2 \pi i)^{n}} \sum_{\lambda}(n)_{\lambda} s_{\lambda}(\mathbf{t}) s_{\lambda}\left(\mathbf{t}^{*}\right),
\end{aligned}
$$

где было использовано то, что в соответствии с (2.1)

$$
(n)_{\lambda}=\prod_{i, j \in \lambda}(n+j-i)=\frac{\Gamma\left(n+1+\lambda_{1}\right) \Gamma\left(n+\lambda_{2}\right) \ldots \Gamma\left(\lambda_{n}\right)}{\Gamma(n+1) \Gamma(n) \ldots \Gamma(1)} .
$$

Таким образом, получается следующий ряд теории возмущений:

$$
\frac{I^{2 M M}\left(n, \mathbf{t}, \mathbf{t}^{*}\right)}{I^{2 M M}(n, 0,0)}=\sum_{\lambda}(n)_{\lambda} s_{\lambda}(\mathbf{t}) s_{\lambda}\left(\mathbf{t}^{*}\right) .
$$

В случае, когда все старшие параметры $t_{j}, t_{j}^{*}$ с $j>2$ обрашаются в нуль (т.е. имеет место случай гауссова матричного интеграла) и $n=1$, этот ряд может быть легко вычислен и приведен к виду (см., например, [15])

$$
\begin{aligned}
& I^{2 M M}\left(1, t_{1}, t_{2}, 0,0, \ldots ; t_{1}^{*}, t_{2}^{*}, 0,0, \ldots\right)=\sum_{m=0}^{\infty} m ! h_{m}(\mathbf{t}) h_{m}\left(\mathbf{t}^{*}\right)= \\
& \quad=\frac{1}{\sqrt{1-4 t_{2} t_{2}^{*}}} \exp \left(\frac{t_{1} t_{1}^{*}+t_{2}\left(t_{1}^{*}\right)^{2}+t_{2}^{*}\left(t_{1}\right)^{2}}{1-4 t_{2} t_{2}^{*}}\right) .
\end{aligned}
$$


5.3. Эрмитова одноматричная модель. Как было показано в работе [18], статистическая сумма эрмитовой одноматричной модели представляет собой тау-функцию одномерной РТ, удовлетворяюшую так называемым условиям Вирасоро. Рассмотрим здесь ряд теории возмушений для этой модели в разложении по функциям Шура. Пусть теперь $M$ - эрмитова матрица размера $N \times N$. Статистическая сумма эрмитовой одноматричной модели с четным потенциалом четвертого порядка имеет вид

$$
Z\left(N, g, g_{4}\right)=\int \exp \left(-N \operatorname{tr}\left(\frac{g}{2} M^{2}+g_{4} M^{4}\right)\right) d M,
$$

где

$$
d M=\prod_{i<k} d \operatorname{Re} M_{i k} d \operatorname{Im} M_{i k} \prod_{i=1}^{n} d M_{i i} .
$$

Выберем нормировку матричного интеграла таким образом, чтобы $Z\left(N, g, g_{4}\right)$ было равно единице при $g_{4}=0$. Вычислим низшие члены ряда теории возмушений для одноматричной модели, используя ряды (5.6) [15]. Сначала рассмотрим статистическую сумму (5.3) двухматричной модели и положим все $t_{k}=0$, кроме $t_{4}$, и все $t_{k}^{*}=0$, кроме $t_{2}^{*}$. Тогда (ср. [19], [20]) известно, что если положить

$$
g_{4}=-4 N^{-1} t_{4}, \quad g=-\left(2 N t_{2}^{*}\right)^{-1},
$$

получим

$$
I^{2 M M}\left(0,0,0, t_{4}, 0, \ldots ; 0, t_{2}^{*}, 0,0, \ldots\right)=Z\left(N, g, g_{4}\right),
$$

а потому

$$
\sum_{\lambda}(N)_{\lambda} s_{\lambda}(\mathbf{t}) s_{\lambda}\left(\mathbf{t}^{*}\right)=\int \exp \left(-N \operatorname{tr}\left(\frac{g}{2} M^{2}+g_{4} M^{4}\right)\right) d M=Z\left(N, g, g_{4}\right) .
$$

ЗАмечАниЕ. Хорошо известно (см., например, [20]), что в соответствии с правилами Фейнмана для одноматричной модели: а) с каждым пропагатором (двойной линией) связан множитель $1 /(N g)$ (равньй $-2 t_{2}^{*}$ в наших обозначениях); б) с каждой четырехточечной вершиной связан множитель $\left(-N g_{4}\right)$ (равный $4 t_{4}$ в наших обозначениях); в) с каждой замкнутой сплошной линией связан множитель $N$. Поэтому можно считать, что в формуле (5.8) множитель $(N)_{\lambda}$ отвечает замкнутым линиям, множители $s_{\lambda}(\mathbf{t})$, $\mathbf{t}=\left(0, t_{2}, 0,0, \ldots\right),-$ пропагаторам, а множители $s_{\lambda}\left(\mathbf{t}^{*}\right)-$ вершинам. Фейнмановские диаграммы, содержашие $k=|\lambda| / 4$ вершин и $2 k=|\lambda| / 2$ пропагаторов, дают следуюший вклад:

$$
\left(N g_{4}\right)^{|\lambda| / 4}(N g)^{-|\lambda| / 2} \sum_{|\lambda|=4 k}(N)_{\lambda} s_{\lambda}(0,0,0,1,0, \ldots) s_{\lambda}(0,1,0, \ldots),
$$

где число $|\lambda|$ равно весу разбиения $\lambda$.

Явное вычисление первых трех неисчезаюших членов (отвечаюших разбиениям весов 0,4 и 8 ) дает тот же ответ, что и вычисление в технике фейнмановских графов (ср. с работой $[20])$ :

$$
Z\left(N, g, g_{4}\right)=1-\frac{g_{4}}{g^{2}}\left(\frac{N^{2}}{2}+\frac{1}{4}\right)+\frac{g_{4}^{2}}{g^{4}}\left(32 N^{4}+320 N^{2}+488\right)+O\left(\frac{g_{4}^{3}}{g^{6}}\right) .
$$




\section{6. ВЫРАЖЕНИЕ ДЛЯ $\ln \tau_{r}\left(n, \mathbf{t}, \mathbf{t}^{*}\right)$}

Напомним, что произведение двух функций Шура можно представить в виде суммы [1]:

$$
s_{\lambda}(\mathbf{t}) s_{\mu}(\mathbf{t})=\sum_{\nu} C_{\lambda \mu}^{\nu} s_{\nu}(\mathbf{t}),
$$

где коэффициенты $C_{\lambda \mu}^{\nu}$ могут быть вычислены по комбинаторным правилам Литтлвуда-Ричардсона [1], а вес $|\nu|$ разбиений в сумме равен сумме весов:

$$
|\nu|=|\lambda|+|\mu|
$$

Записывая (4.6) как $\tau_{r}\left(n, \mathbf{t}, \mathbf{t}^{*}\right)=1+\varepsilon, \varepsilon=\sum_{\lambda \neq 0} r_{\lambda}(n) s_{\lambda}(\mathbf{t}) s_{\lambda}\left(\mathbf{t}^{*}\right)$ и вычисляя логарифм этого выражения, получим

$$
\ln \tau_{r}\left(n, \mathbf{t}, \mathbf{t}^{*}\right)=\sum_{\lambda, \lambda^{\prime} \neq 0} s_{\lambda}(\mathbf{t}) k_{\lambda \lambda^{\prime}}(n) s_{\lambda^{\prime}}\left(\mathbf{t}^{*}\right),
$$

где величины $k_{\lambda \lambda^{\prime}}$ задаются следующими рядами:

$$
\begin{gathered}
k_{\lambda \lambda^{\prime}}(n)=\frac{1}{1} r_{\lambda}(n) \delta_{\lambda \lambda^{\prime}}+\sum_{k>1} \frac{1}{k} \sum_{\lambda_{1}, \ldots, \lambda_{k} \neq 0} r_{\lambda_{1}}(n) \ldots r_{\lambda_{k}}(n) \times \\
\times \sum_{\nu_{1}, \ldots, \nu_{k-2}} C_{\nu_{1}, \ldots, \nu_{k-2}}^{\lambda} \sum_{\nu_{1}^{\prime}, \ldots, \nu_{k-2}^{\prime}} C_{\nu_{1}^{\prime}, \ldots, \nu_{k-2}^{\prime}}^{\lambda^{\prime}}, \\
C_{\nu_{1}, \ldots, \nu_{k-2}}^{\lambda}:=C_{\lambda_{1} \lambda_{2}}^{\nu_{1}} C_{\nu_{1} \lambda_{3}}^{\nu_{2}} \ldots C_{\nu_{k-3} \lambda_{k-1}}^{\nu_{k}} C_{\nu_{k-2} \lambda_{k}}^{\lambda}, \\
C_{\nu_{1}^{\prime}, \ldots, \nu_{k-2}^{\prime}}^{\lambda^{\prime}}:=C_{\lambda_{1} \lambda_{2}}^{\nu_{1}^{\prime}} C_{\nu_{1}^{\prime} \lambda_{3}}^{\nu_{2}^{\prime}} \ldots C_{\nu_{k-3}^{\prime} \lambda_{k-1}^{\prime}}^{\nu_{k-2}^{\prime}} C_{\nu_{k-2}^{\prime} \lambda_{k}^{\prime}}^{\lambda^{\prime}}
\end{gathered}
$$

Первые несколько членов имеют вид

$$
\begin{aligned}
k_{\lambda \lambda^{\prime}}(n)= & \frac{1}{1} r_{\lambda}(n) \delta_{\lambda \lambda^{\prime}}+\frac{1}{2} \sum_{\lambda_{1}, \lambda_{2} \neq 0} r_{\lambda_{1}}(n) r_{\lambda_{2}}(n) C_{\lambda_{1} \lambda_{2}}^{\lambda} C_{\lambda_{1} \lambda_{2}}^{\lambda^{\prime}}+ \\
& +\frac{1}{3} \sum_{\lambda_{1}, \lambda_{2}, \lambda_{3} \neq 0} r_{\lambda_{1}}(n) r_{\lambda_{2}}(n) r_{\lambda_{3}}(n) \sum_{\nu} C_{\lambda_{1} \lambda_{2}}^{\nu} C_{\nu \lambda_{3}}^{\lambda} \sum_{\nu^{\prime}} C_{\lambda_{1} \lambda_{2}}^{\nu^{\prime}} C_{\nu^{\prime} \lambda_{3}}^{\lambda^{\prime}}+\cdots
\end{aligned}
$$

Заметим, что согласно формуле (6.1) каждый член $k_{\lambda \lambda^{\prime}}(n)$ представляется суммой конечного числа слагаемых. Данный результат было бы интересно сравнить с работой [21].

Благодарности. Работа частично поддержана Научным советом Канады по естественным и инженерным наукам (NSERC) и фондом FCAR du Québec, проектом LDRD № 20020006ER "Нестабильные междужидкостные взаимодействия" при Лос-Аламосской национальной лаборатории и грантом РФФИ № 02-02-17382а. 


\section{Список литературы}

[1] I. G. Macdonald. Symmetric Functions and Hall Polynomials. Oxford: Clarendon Press, 1995.

[2] M. Feigin, A. P. Veselov. Internat. Math. Res. Notices. 2002. V. 10. P. 521-545.

[3] А.Ю. Орлов, Д. М. Щербин. ТМФ. 2001. Т. 128. № 1. С. 84-108.

[4] E. Date, M. Jimbo, M. Kashiwara, T. Miwa. Transformation groups for soliton equations. In: Nonlinear Integrable Systems - Classical Theory and Quantum Theory. Proc RIMS, Kioto, 1981. Eds. M. Jimbo, T. Miwa. Singapore: World Scientific, 1983. P. 39-120.

[5] K. Ueno, K. Takasaki. Adv. Stud. Pure Math. 1984. V. 4. P. 1-95.

[6] В.Е. Захаров, С.В. Манаков, С.П. Новиков, Л.П. Питаевский. Теория солитонов: Метод обратной задачи. М.: Наука, 1980.

[7] L. A. Dickey. Soliton Equations and Hamiltonian Systems. Adv. Ser. Math. Phys. V. 12. Singapore-New Jersey-London: World Scientific, 1991.

[8] П. Винтернии, А.Ю. Орлов. ТМФ. 1997. Т. 113. № 2. С. 231-260.

[9] K. Takasaki. Commun. Math. Phys. 1996. V. 181. P. 131-156.

[10] A. Yu. Orlov. Soliton theory, symmetric functions and matrix integrals. nlin.SI/0207030.

[11] T. Takebe. Lett. Math. Phys. 1991. V. 21. P. 77-84.

[12] L.-L. Chau, O. Zaboronsky. Commun. Math. Phys. 1998. V. 196. P. 203-247; hep-th/9711091.

[13] M. Mineev-Weinstein, P. Wiegmann, A. Zabrodin. Phys. Rev. Lett. $2000 . \quad$ V. 84. P. 5106-5109.

[14] J. Harnad, A. Yu. Orlov. Matrix integrals as Borel sums of Schur function expansions. Preprint CRM-2865. Montréal: CRM, 2002; nlin.SI/0209035; In: Symmetry and Perturbation Theory SPT2002. Eds. S. Abenda, G. Gaeta, S. Walcher. Singapore: World Scientific, 2003.

[15] J. Harnad, A. Yu. Orlov. Schur function expansions of matrix integrals. Preprint CRM. Montréal: CRM, 2002.

[16] M. L. Mehta. Random Matrices. San Diego: Academic Press, 1991.

[17] C. Itzykson, J. B. Zuber. J. Math. Phys. 1980. V. 21. P. 411-421.

[18] A. Gerasimov, A. Marshakov, A. Mironov, A. Morozov, A. Orlov. Nucl. Phys. B. 1991. V. 357. P. 565-618.

[19] M. Bertola, B. Eynard, J. Harnad. J. Phys. A. 2003. V. 36. P. 3067-3084; nlin.SI/0204054.

[20] B. Eynard. Random matrices. Preprint Saclay-T01/014, CRM-2708. Paris: Cours de Physique Theorique de Saclay, 2001.

[21] Yu. Klimov, A. Korzh, S. Natanson. From 2D Toda hierarchy to conformal map for domains of Riemann sphere. math.NA/0212361. 